\title{
Les déterminants du choix de carrière des enseignants de la formation professionnelle et leur relation aux caractéristiques sociodémographiques Determinants of Career Choice among Vocational Education Teachers and their Relationship to Socio-Demographic Characteristics \\ Los determinantes de la elección de carrera de los docentes de la formación profesional y su relación con las características sociodemográficas
}

Yannick D’Ascoli et Jean-Louis Berger

Volume 15, numéro 2, 2012

URI : https://id.erudit.org/iderudit/1018455ar

DOI : https://doi.org/10.7202/1018455ar

Aller au sommaire du numéro

Éditeur(s)

Faculté d'éducation, Université de Sherbrooke

ISSN

1911-8805 (numérique)

Découvrir la revue

Citer cet article

D’Ascoli, Y. \& Berger, J.-L. (2012). Les déterminants du choix de carrière des enseignants de la formation professionnelle et leur relation aux caractéristiques sociodémographiques. Nouveaux cahiers de la recherche en éducation, 15(2), 1-33. https://doi.org/10.7202/1018455ar
Résumé de l'article

Constatant qu'une pénurie d'enseignants se profile dans de nombreux pays occidentaux, cette étude, menée en Suisse, se focalise sur les motivations des enseignants de ce pays et leurs perceptions du métier. Des analyses de pistes causales ont été menées à partir de données recueillies au moyen d'un questionnaire construit à partir du modèle théorique Factors Influencing Teaching Choice. Les résultats obtenus mettent en évidence l'importance des motivations intrinsèques et sociales dans le choix de devenir enseignant ainsi que certaines différences dues aux caractéristiques sociodémographiques. Finalement, des implications concernant le recrutement des enseignants sont proposées. 


\section{Les déterminants du choix de carrière des enseignants de la formation professionnelle et leur relation aux caractéristiques sociodémographiques}

\section{Yannick D'Ascoli}

Institut fédéral des hautes études en formation professionnelle, Lausanne

\section{Jean-Louis Berger}

Institut fédéral des hautes études en formation professionnelle, Lausanne

\section{Les déterminants du choix de carrière des enseignants de la formation professionnelle et leur relation aux caractéristiques sociodémographiques}

\section{Résumé}

Constatant qu'une pénurie d'enseignants se profile dans de nombreux pays occidentaux, cette étude, menée en Suisse, se focalise sur les motivations des enseignants de ce pays et leurs perceptions du métier. Des analyses de pistes causales ont été menées à partir de données recueillies au moyen d'un questionnaire construit à partir du modèle théorique Factors Influencing Teaching Choice. Les résultats obtenus mettent en évidence l'importance des motivations intrinsèques et sociales dans le choix de devenir enseignant ainsi que certaines différences dues aux caractéristiques sociodémographiques. Finalement, des implications concernant le recrutement des enseignants sont proposées.

Mots clés: formation professionnelle, FIT-Choice, transition professionnelle, motivation à enseigner, perceptions de l'enseignement 


\title{
Determinants of Career Choice among Vocational Education Teachers and their Relationship to Socio-Demographic Characteristics
}

\begin{abstract}
In light of the shortage of teachers arising in numerous Western countries, this study, led in Switzerland, focuses on the motivations of teachers in this country and their perceptions of the occupation. Analyses of causal avenues were performed based on data collected using a questionnaire that was developed using the theoretical model Factors Influencing Teaching Choice. The results obtained underline the importance of intrinsic and social motivations in the choice to become a teacher, as well as certain differences due to socio-demographic characteristics. Finally, the article proposes implications for teacher recruitment.
\end{abstract}

Key words: vocational education, FIT-Choice, employment transition, motivation to teach, perceptions of teaching

\section{Los determinantes de la elección de carrera de los docentes de la formación profesional y su relación con las características sociodemográficas}

\section{Resumen}

Constatando que en numerosos países occidentales existe una escasez de profesores, el presente estudio realizado en Suiza, se focaliza en las motivaciones de los docentes de este país y sus percepciones sobre el oficio. Se llevaron a cabo análisis de pistas causales utilizando datos recogidos por medio de un cuestionario construido a partir del modelo teórico Factors Influencing Teaching Choice. Los resultados obtenidos colocan en evidencia la importancia de las motivaciones intrínsecas y sociales como factores presentes en la opción de convertirse en docente, al igual que algunas diferencias atribuidas a las características sociodemográficas. Finalmente, se proponen algunas implicancias referentes a la selección de los docentes.

Palabras clave: formación, FIT-Choice, transición profesional, motivación para enseñar, percepciones de la enseñanza 


\section{Introduction}

\subsection{Problématique}

La formation professionnelle occupe une place prépondérante dans le milieu éducatif suisse. Selon l'Office Fédéral de la Statistique (Office fédéral de la statistique suisse, 2010a), c'est la voie la plus fréquemment choisie par les jeunes (70 \%) après leur scolarité obligatoire. De plus, dans le contexte international, la formation professionnelle est considérée comme prépondérante au niveau économique et social (Achtenhagen et Grubb, 2001): près des deux tiers des employés des pays occidentaux ont acquis leurs compétences et connaissances non pas dans un milieu académique, mais dans une formation professionnelle dans le cadre de professions exigeant un niveau de spécialisation qu'il n'est pas possible d'atteindre par la voix universitaire (Grollmann, 2008).

En dépit de son importance, le milieu de la formation professionnelle n'échappe cependant pas à la problématique de la pénurie d'enseignants observée aux divers échelons éducatifs en Suisse et dans nombre de pays occidentaux (Centre suisse de coordination pour la recherche en éducation, 2010; Eurydice, 2009; OCDE, 2005). Ce manque d'effectifs trouverait son origine dans une certaine perte d'attractivité du métier d'enseignant (Conférence suisse des directeurs cantonaux de l'instruction publique, 2008; Gavillet-Mentha, 2011; Jellab, 2008; Maroy, 2008; OCDE, 2005) ainsi que dans l'augmentation de l'âge moyen des enseignants. En effet, le pourcentage des enseignants de la formation professionnelle âgés de plus de 50 ans a évolué, de $32 \%$ en 1999 à 39,5\% en 2009 (Office fédéral de la statistique suisse, 2010b), suggérant une possible pénurie dans le futur. Ce grand nombre d'enseignants qui prendra sa retraite durant les 15 prochaines années appartient à la génération dite des baby-boomers. Leur remplacement constitue un défi dans de multiples domaines professionnels.

Relativement peu de recherches se sont intéressées à la population des enseignants en formation professionnelle pour qui l'enseignement consiste, pour la grande majorité, en une deuxième carrière après celle exercée dans leur domaine de formation initiale. 
Cependant, un certain intérêt pour cette thématique est apparu ces dernières années dans la littérature francophone en sociologie de l'éducation (Jellab, 2008) et chez un certain nombre de chercheurs québécois (Balleux et Perez-Roux, 2011; Beaucher et Balleux, 2010). Deschenaux et Roussel (2011) qui mettent en évidence l'importance des expériences accumulées par les enseignants lors de leur première carrière professionnelle dans le vécu de leur transition vers l'enseignement. Effectivement, comme le souligne Balleux (2011), les enseignants de la formation professionnelle sont particulièrement confrontés au phénomène, largement traité dans les études portant sur les enseignants en général, du «choc de la réalité» lors de la période de formation ainsi qu'au début de l'activité professionnelle, à cause des nombreux nouveaux défis auxquels ils doivent répondre (confrontation avec les élèves, manque de soutien à disposition, disponibilités, complexité, baisse de salaire). Berger et D'Ascoli (2012a) ont également étudié l'influence de la première carrière, cette fois-ci sur les motivations à devenir enseignants de la formation professionnelle. Bien que se focalisant sur un échantillon restreint d'enseignants du niveau primaire, l'étude de Duschesne (2011) apporte à ce titre un éclairage précieux en soulignant que le changement de carrière naîtrait d'une insatisfaction et de besoins non comblés dans le cadre de l'exercice de la profession précédente et que les motivations amenant à l'enseignement seraient liées à des facteurs sociaux (contribuer à la société, donner un sens à sa vie, travailler avec la jeunesse).

L'étude des motivations et perceptions des enseignants, au vu de leur influence considérable sur l'engagement et l'investissement dans la profession (Berger et D'Ascoli, 2011; Huberman, 1989; Müller, Alliata et Benninghoff, 2009; Sinclair, 2008; Watt et Richardson, 2007), pourrait également apporter de nouvelles perspectives dans les stratégies de recrutement et des offres de formation. Les acteurs de la formation professionnelle pourraient à l'avenir prendre davantage en considération les attentes des enseignants lors de l'engagement de personnel et la planification des cursus de formation. Cette recherche s'inscrit dans l'optique de ces diverses implications.

Cette étude s'intéresse également aux associations entre les caractéristiques sociodémographiques et les déterminants du choix de devenir enseignant. Cette question se justifie d'autant plus que le public enseignant dans la formation professionnelle suisse se distingue par une hétérogénéité démographique, professionnelle (profession initiale), 
culturelle, sociale et par son taux d'activité en tant qu'enseignant. Dans l'enseignement professionnel en Suisse, il est en effet courant qu'une personne exerce la fonction d'enseignant tout en continuant d'exercer sa profession de terrain. Ainsi, à titre d'exemple, un électricien peut-il enseigner une dizaine d'heures dans une école professionnelle et conduire en parallèle une petite entreprise de réparations de systèmes électriques chez des particuliers. En contraste, d'autres enseignants ont choisi d'effectuer un changement de carrière; ils transitent ainsi de l'exercice d'une profession à son enseignement. Il est légitime de se demander si ces deux types d'enseignants de la formation professionnelle choisissent le métier pour les mêmes raisons. Concrètement, notre recherche questionne le lien éventuel entre les caractéristiques des enseignants telles que l'appartenance à différentes régions linguistiques ou différents domaines professionnels et l'importance de diverses motivations à devenir enseignant ainsi que leurs perceptions de la profession. Pour ce faire, nous avons adopté un modèle théorique qui englobe les différentes motivations et perceptions liées à l'entrée dans la profession enseignante.

\subsection{Le modèle FIT-Choice et son adaptation}

Dans une volonté d'étudier le choix du métier d'enseignant, Watt et Richardson (2007, 2008) et Richardson et Watt $(2006,2010)$ ont développé un modèle théorique nommé Factors Influencing Teaching Choice (FIT-Choice). Le modèle est basé sur la théorie Expectancy-Value utilisée pour l'étude des motivations dans les choix académiques et de carrière (Eccles, Adler, Futterman, Goff, Kaczala, Meece et Midgley, 1983). Le modèle met en évidence deux types de perception qui détermineraient les choix dans un certain domaine: premièrement les attentes de réussite, c'est-à-dire la perception par l'individu de ses propres capacités (Expectancy) et deuxièmement la valeur (caractéristiques) subjective positive ou négative attribuée à la tâche. Il considère différents types de motivations notamment intrinsèques, d'utilité personnelle, d'utilité sociale, ainsi que les perceptions de ses propres capacités. Enfin d'autres variables prédictives d'ordre social, culturel ou lié aux expériences passées personnelles sont également considérées. 
Le modèle FIT-Choice représente une tentative de regroupement des thématiques émergeant des recherches sur les déterminants du choix de carrière des enseignants. Il tente ainsi de combler le manque observé par Watt et Richardson (2007) au niveau des modèles théoriques et des instruments de mesure valides dans le domaine du choix de carrière dans l'enseignement (Ibid.). Le modèle FIT-Choice considère d'une part, les principaux facteurs de motivation amenant au choix de l'enseignement et d'autre part, les perceptions liées à ce métier.

Concernant les motivations, le modèle comporte quatre composantes: perceptions de soi, carrière par défaut, valeurs et influences de socialisation. Dans chacune de ces composantes ont été définies plusieurs dimensions (14 au total). Pour la première composante concernant les perceptions de soi, une unique dimension, «perception des capacités à enseigner», considère la perception que l'individu a de ses propres capacités pour mener des activités d'enseignement. La deuxième composante, carrière par défaut, souligne le choix professionnel par manque de possibilité d'exercer la profession que les personnes auraient privilégiée. En d'autres termes, il s'agit de l'idée que l'enseignement est un choix réalisé à défaut de mieux. Étant donné que nos participants viennent à l'enseignement dans le contexte d'un second choix professionnel, cette composante n'avait pas lieu d'être conservée et nous l'avons remplacée par une composante intitulée «opportunité». Cette dernière comporte la dimension de «choix par opportunité», qui souligne la venue à l'enseignement, car l'occasion s'est présentée (motivation passive) plutôt qu'à la suite d'une recherche d'emploi volontaire (Nägele et Bestvater, 2012; Deschenaux et Roussel, 2008). La troisième composante, valeur, comporte neuf dimensions. Tout d'abord, «valeur intrinsèque de la carrière» reprend l'idée de l'intérêt pour la profession. Ensuite, quatre dimensions, «sécurité de l'emploi», «transférabilité de l'emploi», «temps pour la famille» et «facilité de l'emploi» représentent autant de facettes d'utilité personnelle. La dimension «transférabilité de l'emploi», qui évalue l'enseignement comme permettant de travailler à l'étranger et de déterminer le lieu de domicile, n'était pas adaptée au contexte de notre recherche et n'a ainsi pas été retenue. Puis, quatre autres dimensions: «influencer le futur des enfants/adolescents», «promotion de l'équité sociale», «réaliser une contribution sociale» et «travailler avec des enfants/adolescents» composent les facettes d'utilité sociale. Finalement, trois dimensions forment la composante influences de socialisation: «expériences passées dans 
l'enseignement et l'apprentissage» qui réfère à l'influence du vécu de la scolarité dans le choix du métier d'enseignant, «influences sociales» et «dissuasion sociale» (pouvant provenir toutes deux de la famille, d'amis ou de collègues de travail).

Pour ce qui a trait aux perceptions du métier d'enseignant, quatre dimensions concernent: les conditions salariales (salaire), l'exigence de la tâche (forte demande), le niveau de connaissances requis (carrière d'expert), l'image de la profession et l'état moral des enseignants (statut social et moral des enseignants).

Le tableau 1 reprend les différentes échelles du modèle FIT-Choice adapté, leurs définitions ainsi qu'un exemple d'item dans chaque échelle. 
Tableau 1

Description des dimensions provenant du modèle adapté FIT-Choice

\begin{tabular}{|c|c|c|}
\hline Dimensions & Définition & Exemple d'item \\
\hline \multicolumn{3}{|l|}{ Perceptions de soi } \\
\hline $\begin{array}{l}\text { Perception des capacités à enseigner } \\
\text { Opportunité }\end{array}$ & Perception des capacités pour enseigner & J'ai de bonnes compétences pour enseigner. \\
\hline $\begin{array}{l}\text { Choix par opportunité (nouvelle } \\
\text { échelle) }\end{array}$ & Choix de l'enseignement par opportunité & L'opportunité d'enseigner s'est présentée. \\
\hline \multicolumn{3}{|l|}{ Valeurs } \\
\hline $\begin{array}{l}\text { Valeur intrinsèque de la carrière } \\
\text { Utilité sociale }\end{array}$ & Choix de l'enseignement par motivation intrinsèque & J'aime enseigner. \\
\hline $\begin{array}{l}\text { Influencer le futur des } \\
\text { enfants/adolescents }\end{array}$ & $\begin{array}{l}\text { Choix de l'enseignement pour transmettre des valeurs aux } \\
\text { jeunes }\end{array}$ & $\begin{array}{l}\text { L'enseignement me permet de transmettre } \\
\text { certaines valeurs aux jeunes. }\end{array}$ \\
\hline Améliorer l'équité sociale & $\begin{array}{l}\text { Choix de l'enseignement pour aider et élever les } \\
\text { ambitions des jeunes défavorisés }\end{array}$ & $\begin{array}{l}\text { L’enseignement me permet d'élever les } \\
\text { ambitions des jeunes défavorisés. }\end{array}$ \\
\hline Réaliser une contribution sociale & Choix de l'enseignement comme contribution sociale & $\begin{array}{l}\text { L'enseignement me permet de rendre un } \\
\text { service à la société. }\end{array}$ \\
\hline Travail avec des enfants/adolescents & $\begin{array}{l}\text { Choix de l'enseignement pour travailler et aider les } \\
\text { jeunes }\end{array}$ & Je souhaite travailler avec des jeunes. \\
\hline \multicolumn{3}{|l|}{ Utilité personnelle } \\
\hline Sécurité de l'emploi & $\begin{array}{l}\text { Choix de l'enseignement comme métier sûr en termes de } \\
\text { stabilité de l'emploi et des possibilités de carrière }\end{array}$ & L’enseignement est un métier sûr. \\
\hline Temps pour la famille & $\begin{array}{l}\text { Choix de l'enseignement pour pouvoir consacrer plus de } \\
\text { temps à la famille et améliorer sa qualité de vie }\end{array}$ & $\begin{array}{l}\text { Les heures d'enseignement sont en } \\
\text { adéquation avec les responsabilités } \\
\text { familiales. }\end{array}$ \\
\hline
\end{tabular}




\begin{tabular}{|c|c|c|}
\hline Dimensions & Définition & Exemple d'item \\
\hline Facilité de l'emploi & $\begin{array}{l}\text { Choix de l'enseignement pour ses jours de travail courts } \\
\text { et ses longues vacances }\end{array}$ & $\begin{array}{l}\text { En tant qu'enseignant, j’ai de longues } \\
\text { vacances. }\end{array}$ \\
\hline \multicolumn{3}{|l|}{ Influences de socialisation } \\
\hline $\begin{array}{l}\text { Expériences passées dans } \\
\text { l'enseignement et l'apprentissage }\end{array}$ & $\begin{array}{l}\text { Perception de la qualité de certains enseignants en tant } \\
\text { qu'ancien élève }\end{array}$ & J'ai eu des enseignants qui m'ont inspiré. \\
\hline Influences sociales & $\begin{array}{l}\text { Perception des encouragements et persuasions de } \\
\text { l'entourage à devenir enseignant }\end{array}$ & $\begin{array}{l}\text { Mes amis pensaient que je devrais devenir } \\
\text { enseignant. }\end{array}$ \\
\hline Dissuasion sociale & $\begin{array}{l}\text { Perception des dissuasions de l'entourage à devenir } \\
\text { enseignant }\end{array}$ & $\begin{array}{l}\text { Vous a-t-on dit que l'enseignement n'était } \\
\text { pas un bon choix de carrière? }\end{array}$ \\
\hline \multicolumn{3}{|l|}{$\begin{array}{c}\text { Perceptions de la carrière } \\
\text { enseignante }\end{array}$} \\
\hline Demandes de l'emploi & & \\
\hline Carrière d'expert & $\begin{array}{l}\text { Perception de l'enseignement comme requérant une } \\
\text { expertise de haut niveau }\end{array}$ & $\begin{array}{l}\text { Pensez-vous que l'enseignement requiert } \\
\text { une expertise de haut niveau? }\end{array}$ \\
\hline Forte demande & Perception d'une lourde charge de travail & $\begin{array}{l}\text { Pensez-vous que les enseignant-e-s soient } \\
\text { soumis à une lourde charge de travail? }\end{array}$ \\
\hline $\begin{array}{l}\text { Bénéfices de l'emploi } \\
\text { Statut social et moral des } \\
\text { enseignants }\end{array}$ & $\begin{array}{l}\text { Perception de l'enseignement comme un métier } \\
\text { socialement et professionnellement valorisé }\end{array}$ & $\begin{array}{l}\text { Pensez-vous que l'enseignement soit perçu } \\
\text { comme un métier ayant un haut statut? }\end{array}$ \\
\hline Salaire & $\begin{array}{l}\text { Perception de l'enseignement comme une activité bien } \\
\text { rémunérée }\end{array}$ & $\begin{array}{l}\text { Pensez-vous que l'enseignement soit un } \\
\text { métier bien rémunéré? }\end{array}$ \\
\hline
\end{tabular}


Les déterminants du choix de carrière des enseignants de la formation professionnelle...

Une description plus détaillée du modèle FIT-Choice est disponible dans Watt et Richardson (2007) ainsi que dans Berger et D'Ascoli (2011), respectivement en anglais et en français.

\subsection{Objectifs de l'étude et questions de recherche}

$\mathrm{Au}$ vu de l'importance de la place qu'occupe la formation professionnelle en Suisse et considérant le phénomène de la pénurie d'enseignants dans de nombreux pays occidentaux, notre étude cherche à analyser les motivations et les perceptions des enseignants en formation professionnelle (deuxième carrière) concernant leur métier ainsi que leurs relations avec les caractéristiques démographiques des enseignants. Les analyses et la discussion des résultats seront menées sur la base des questions de recherche suivantes:

1) Quels sont les déterminants du choix de carrière les plus importants chez les enseignants en formation professionnelle?

2) Dans quelle mesure la prépondérance de ces déterminants dépend-elle des caractéristiques démographiques des enseignants de la formation professionnelle?

\section{Méthodologie}

\subsection{Participants}

L'échantillon est composé de 483 enseignants de la formation professionnelle en Suisse $^{1}$ qui suivent une formation en cours d'emploi, dont 253 exercent l'enseignement à titre accessoire et 230 à titre principal. Ils sont déjà actifs dans l'enseignement, généralement depuis plusieurs années, au moment de leur entrée en

\footnotetext{
${ }^{1}$ En Suisse, la formation professionnelle se décline principalement sous un mode de type dual: les apprentis suivent un cursus qui se déroule alternativement à l'école (un ou deux jours par semaine) et en entreprise (entre trois et quatre jours par semaine). Plus de détails concernant le système de formation professionnelle suisse peuvent être consultés dans divers rapports (Dubs, 2006, Office fédéral de la formation professionnelle et de la technologie, 2011).
} 
Les déterminants du choix de carrière des enseignants de la formation professionnelle...

formation. Pour le premier groupe, l'enseignement est une activité auxiliaire, c'est-àdire à un taux d'occupation inférieur à $50 \%$. La majorité d'entre eux continue de travailler parallèlement dans leur domaine de formation initiale. Leur formation dure 300 heures et a comme objectif de leur fournir les connaissances pédagogiques de base. Les enseignants à titre principal suivent une formation de 1800 heures sur deux ou trois ans ce qui représente une extension de la formation de 300 heures. Ces derniers ont décidé de changer de carrière et de se consacrer exclusivement à leur nouvelle profession. Les étudiants de cette filière doivent par conséquent acquérir des connaissances pédagogiques plus avancées concernant, par exemple, le développement et l'évaluation des compétences ou l'approche des processus interactionnels dans divers contextes éducatifs.

Les enseignants, provenant des deux principales régions linguistiques suisses, sont en majorité de langue maternelle allemande $(n=328,67,9 \%)$, les francophones représentant environ un tiers de l'échantillon $(n=155,32,1 \%)$. Près des deux tiers d'entre eux sont des hommes (65\%). L'âge des répondants varie entre 22 et 58 ans (moyenne de 40 ans et 8 mois et écart-type de 7 ans et 2 mois), la moitié de l'échantillon se situant dans la tranche des 35 à 46 ans. L'âge moyen des personnes devenant enseignantes en formation professionnelle est donc relativement avancé (en comparaison aux enseignants de l'école obligatoire), ce qui implique une carrière relativement courte dans le domaine (une vingtaine d'années). Cela s'explique évidemment par le fait que l'entrée dans l'enseignement professionnel constitue un deuxième choix de carrière.

Conséquence d'une absence de pratiques uniformes à ce niveau, le nombre d'années d'expérience dans l'enseignement précédant l'arrivée en formation varie passablement entre les participants selon le canton et l'établissement scolaire. Si la moyenne est d'un peu plus de 5 ans ( 5 ans et 3 mois, écart-type de 4 ans et 9 mois), une grande majorité des personnes questionnées (74,6\%) va se former durant leurs six premières années d'expérience dans l'enseignement alors qu'environ $10 \%$ de l'échantillon enseignent depuis déjà plus de 10 ans. Sur ce point, ce public se distingue des enseignants en formation générale (par exemple secondaire) qui entrent 
Les déterminants du choix de carrière des enseignants de la formation professionnelle...

classiquement en formation à l'enseignement directement à la suite de leurs études universitaires.

Avant leurs débuts dans l'enseignement, certains enseignants de la formation professionnelle peuvent être amenés à s'occuper d'apprentis. Le nombre d'années dévolues à cette tâche varie fortement entre les participants: un peu plus d'un tiers $(36,3 \%)$ n'a jamais eu d'apprentis à charge alors que les autres ont entre une et quarante années d'expérience. À partir des réponses recueillies concernant le diplôme obtenu le plus élevé, les participants ont été classifiés en cinq niveaux de formation différents (provenant de l'International Standard Classification of Education, ISCED): «3A, maturité professionnelle» $(\mathrm{n}=37)$, «3B formation professionnelle/ apprentissage» $(\mathrm{n}=5)$, «5A, haute école spécialisée» $(\mathrm{n}=130)$, «5B, école professionnelle supérieure» $(\mathrm{n}=302)$ et $« 6$, doctorat» $(\mathrm{n}=8)$. Dans une deuxième étape, nous avons regroupé les niveaux $3 \mathrm{a}$ et $3 \mathrm{~b}$ en une caractéristique démographique nommée «niveau de formation professionnelle inférieure», le niveau $5 \mathrm{~b}$ en «niveau de formation professionnelle supérieure» et les niveaux $5 \mathrm{a}$ et 6 en «niveau de formation académique».

$\mathrm{Au}$ niveau familial, 378 participants sont mariés ou en concubinat alors que 104 sont célibataires (1 réponse manquante). Une large majorité de l'échantillon $(69,4 \%)$ a un ou plusieurs enfants en bas âge (moins de 6 ans) et un peu plus de la moitié $(51,3 \%)$ a un ou des enfants plus âgés.

\subsection{Matériel et procédure}

Les enseignants ont été invités à remplir, de façon anonyme, un questionnaire (version électronique ou papier selon les infrastructures informatiques disponibles) durant une période de cours dans le cadre de leur formation. Cette stratégie nous a permis d'atteindre, à l'exception des personnes absentes le jour de notre passage, tous les enseignants en formation à l'Institut fédéral des hautes études en formation professionnelle des régions francophones et alémaniques de Suisse au printemps 2010 et d'obtenir ainsi un taux de réponse extrêmement élevé et un échantillon représentatif. La participation à l'enquête était volontaire; avant de remplir le 
Les déterminants du choix de carrière des enseignants de la formation professionnelle...

questionnaire, les participants ont été informés des objectifs et étapes de l'enquête ainsi que de la structure du questionnaire par un des collaborateurs de l'équipe de recherche qui est resté présent durant toute la durée de la récolte des données afin de répondre aux questions des participants.

\subsubsection{Caractéristiques sociodémographiques}

Les participants étaient invités à répondre à une série de questions portant sur leurs caractéristiques sociodémographiques (description présentée ci-dessus), soit la région linguistique, qui impliquerait également une certaine différence de culture, l'âge, le taux d'activité (activité à titre principal ou accessoire), le sexe, le statut conjugal, le nombre d'enfants de moins et de plus de 6 ans, le niveau de formation ainsi que le nombre d'années d'expérience dans l'encadrement d'apprentis et en tant qu'enseignant.

\subsubsection{Facteurs influençant le choix de devenir enseignant: motivation du choix de l'enseignement et perceptions de la profession}

Les différentes échelles du modèle FIT-Choice ont été traduites de l'anglais à l'allemand et au français (la traduction en français est proposée en annexe). Elles ont également été adaptées au contexte de l'étude, notamment par le remplacement dans certains items des termes «enfants et adolescents» par «jeunes», plus en adéquation avec l'âge (16 à plus de 20 ans) des personnes en formation.

Pour chaque item du questionnaire, les participants devaient fournir une réponse sur une échelle de type Likert à sept niveaux allant pour les motivations de 1 «pas du tout important» à 7 «extrêmement important» et de 1 «non, pas du tout» à 7 «oui, tout à fait» pour ce qui est des perceptions de la profession enseignante.

Un prétest de l'enquête, auprès d'un échantillon de 144 enseignants (110 provenant de la région alémanique et 34 de la région francophone) qui avaient récemment achevé leur cursus, a été mené afin de vérifier la qualité de la traduction et 
Les déterminants du choix de carrière des enseignants de la formation professionnelle...

la pertinence ainsi que l'adéquation des items. Afin de valider les traductions du questionnaire FIT-Choice, les réponses de l'échantillon complet $(\mathrm{N}=483)$ ont été soumises à des analyses factorielles confirmatoires ${ }^{2}$ multigroupe, puis la consistance interne des échelles a été estimée par l'alpha de Cronbach (Berger et D'Ascoli, 2012a, $2012 b)$.

\subsection{Analyse des données}

Dans un premier temps, nous avons testé les différences entre les moyennes afin de déterminer les facteurs rapportés comme les plus importants dans le choix de carrière des enseignants de la formation professionnelle. Dans un second temps, nous avons analysé, à l'aide d'un modèle d'équations structurales ${ }^{3}$, les relations entre les caractéristiques sociodémographiques des enseignants et l'importance qu'ils accordaient aux divers déterminants du choix de carrière ainsi que leurs perceptions de la profession enseignante.

\section{Résultats}

\subsection{Importance relative des facteurs influençant le choix d'une carrière enseignante}

Afin de tester s'il existe des variations significatives des moyennes dans l'importance des motivations ainsi que dans le degré d'accord avec les perceptions,

\footnotetext{
${ }^{2}$ Les analyses factorielles confirmatoires ont été réalisées avec le logiciel Mplus 5.0 (Muthén et Muthén, 2007). Les modèles ont été estimés en utilisant une procédure d'estimation robuste (Maximum Likelihood Robust, MLR) quant aux déviations de la normalité dans les distributions. L'indice $\chi^{2}$ a été corrigé selon la procédure de Satorra-Bentler (Satorra et Bentler, 2001). La proportion de données manquantes, au niveau des items, est inférieure à $5 \%$, ce qui est considéré comme acceptable (Tabachnick et Fidell, 2007). De ce fait, toutes les analyses ont été conduites selon la procédure Full Information Maximum Likelihood (FIML) qui est la méthode la plus efficace pour estimer des modèles d'équations structurales en présence d'un taux raisonnable de données manquantes (Enders, 2006).

${ }^{3}$ Le modèle d'équations structurales a également été réalisé avec le logiciel Mplus 5.0 en utilisant l'estimateur MLR et la procédure FIML. De plus, l'erreur de mesure dans les scores a été fixée en utilisant la formule (1 - fidélité)*variance (Bollen, 1989). Ces corrections permettent de tenir compte de la fidélité des scores et d'obtenir ainsi des paramètres plus précis.
} 
Les déterminants du choix de carrière des enseignants de la formation professionnelle...

des analyses de variance à mesures répétées, suivies de comparaisons multiples des moyennes (avec correction Bonferroni), ont été réalisées sur les participants qui ont répondu à tous les items $(\mathrm{N}=479)$. Les moyennes qui ne diffèrent pas significativement sont indiquées par des lettres identiques dans le tableau 2. Ce tableau récapitule également les différentes échelles, la consistance interne estimée par l'alpha de Cronbach, les statistiques descriptives ainsi que les corrélations entre les échelles FIT-Choice. 
Les déterminants du choix de carrière des enseignants de la formation professionnelle...

Tableau 2

Statistiques descriptives des échelles formées sur la base des analyses factorielles confirmatoires et corrélations entre les échelles des déterminants du choix de carrière

\begin{tabular}{|c|c|c|c|c|c|c|c|c|c|c|c|c|c|c|c|c|c|c|}
\hline & & $\alpha \mathrm{FR}$ & $\alpha \mathrm{DE}$ & M & ET & $\begin{array}{l}\text { Étendue } \\
\text { observée }\end{array}$ & 1 & 2 & 3 & 4 & 5 & 6 & 7 & 8 & 9 & 10 & 11 & 12 \\
\hline & Perceptions de soi & & & & & & & & & & & & & & & & & \\
\hline 1 & $\begin{array}{l}\text { Perception des capacités à } \\
\text { enseigner }\end{array}$ & .85 & .81 & $5.46^{b}$ & 0.97 & $1-7$ & 1 & & & & & & & & & & & \\
\hline & Opportunité & & & & & & & & & & & & & & & & & \\
\hline 2 & Choix par opportunité & .64 & .86 & $4.38^{\mathrm{d}}$ & 1.20 & $1-7$ & -.01 & 1 & & & & & & & & & & \\
\hline & Valeurs & & & & & & & & & & & & & & & & & \\
\hline 3 & $\begin{array}{l}\text { Valeur intrinsèque de la } \\
\text { carrière }\end{array}$ & .80 & .72 & $5.94^{\mathrm{a}}$ & 0.89 & $2-7$ & .60 & -.06 & 1 & & & & & & & & & \\
\hline 4 & Utilité personnelle & .84 & .86 & $2.45^{\mathrm{e}}$ & 0.79 & $1-5.50$ & .10 & .14 & .01 & 1 & & & & & & & & \\
\hline 5 & Utilité sociale & .91 & .90 & $5.00^{\mathrm{c}}$ & 1.08 & $1.39-7$ & .42 & .09 & .51 & .12 & 1 & & & & & & & \\
\hline & $\begin{array}{l}\text { Influences de } \\
\text { socialisation } \\
\text { Expériences passées dans }\end{array}$ & & & & & $1-7$ & & & & & & & & & & & & \\
\hline 6 & $\begin{array}{l}\text { l'enseignement et } \\
\text { l'apprentissage }\end{array}$ & .81 & .87 & $4.39^{\mathrm{d}}$ & 1.56 & & .30 & .07 & .24 & .12 & .29 & 1 & & & & & & \\
\hline 7 & Influences sociales & .87 & .79 & $2.65^{\mathrm{e}}$ & 1.60 & $1-7$ & .19 & .22 & .15 & .21 & .26 & .29 & 1 & & & & & \\
\hline 8 & Dissuasion sociale & .82 & .72 & $2.52^{\mathrm{e}}$ & 1.42 & $1-7$ & .01 & .03 & -.02 & .14 & .08 & .09 & .18 & 1 & & & & \\
\hline
\end{tabular}


Les déterminants du choix de carrière des enseignants de la formation professionnelle...

\begin{tabular}{|c|c|c|c|c|c|c|c|c|c|c|c|c|c|c|c|c|c|c|}
\hline & & $\alpha \mathrm{FR}$ & $\alpha \mathrm{DE}$ & M & ET & $\begin{array}{l}\text { Étendue } \\
\text { observée }\end{array}$ & 1 & 2 & 3 & 4 & 5 & 6 & 7 & 8 & 9 & 10 & 11 & 12 \\
\hline & $\begin{array}{l}\text { Perceptions de la carrière } \\
\text { enseignante }\end{array}$ & & & & & & & & & & & & & & & & & \\
\hline 10 & Forte demande & .55 & .69 & $5.53^{\mathrm{B}}$ & 0.96 & $1.33-7$ & .18 & .06 & .15 & .00 & .19 & .10 & .02 & -.05 & .34 & 1 & & \\
\hline 11 & $\begin{array}{l}\text { Statut social et moral des } \\
\text { enseignants }\end{array}$ & .87 & .86 & $4.31^{\mathrm{D}}$ & 1.03 & $1-7$ & .22 & .08 & .26 & -.08 & .27 & .17 & .18 & -.09 & .35 & .14 & 1 & \\
\hline 12 & Salaire & .95 & .96 & $4.72^{\mathrm{C}}$ & 1.38 & $1-7$ & .12 & .07 & .13 & -.00 & .18 & .12 & .07 & -.06 & -.16 & .04 & .34 & 1 \\
\hline
\end{tabular}

Note: pour toutes les échelles, l'étendue possible est $1-7$. Les corrélations $=|.09|$ sont significatives à $p<.05$; les corrélations $\geq|.12|$ sont significatives à $p<.01$ et les corrélations $\geq|.15|$ sont significatives à $p<.001$ (2-tailed). 
Les déterminants du choix de carrière des enseignants de la formation professionnelle...

Concernant l'importance des motivations, le test de Mauchly indique que le postulat de sphéricité est violé $\left(\chi^{2}(27)=623.985, p<.001\right)$. Par conséquent, les degrés de liberté ont été corrigés selon l'estimation de sphéricité de GreenhouseGeisser $(\varepsilon=.782)$. Les résultats indiquent que l'importance varie en fonction des motivations, $\mathrm{F}(5.472,2615.645)=733.5, p<.001, \eta^{2}=.605$. Les comparaisons multiples des moyennes indiquent que la valeur intrinsèque est jugée comme le facteur significativement plus important que tous les autres. La perception des aptitudes à enseigner est ensuite significativement plus importante que l'utilité sociale. Viennent ensuite la qualité des expériences en tant qu'élèves et opportunité puis les influences sociales, la dissuasion et finalement l'utilité personnelle.

Pour ce qui est du degré d'accord concernant les perceptions du métier d'enseignant, le test de Mauchly indique que le postulat de sphéricité est violé $\left(\chi^{2}(5)=\right.$ $141.912, p<.001)$ ce qui a amené à une correction des degrés de liberté selon l'estimation de sphéricité de Greenhouse-Geisser $(\varepsilon=.831)$. L'importance varie en fonction des perceptions, $\mathrm{F}(2.493,1191.568)=261.923, p<.001, \eta^{2}=.354$. Les comparaisons multiples des moyennes indiquent que les enseignants sont significativement le plus en accord avec le postulat que l'enseignement est une carrière de spécialiste. Viennent ensuite la charge de travail puis la rémunération. Enfin, les enseignants sont significativement le moins en accord avec l'idée selon laquelle le statut professionnel des enseignants est élevé.

\subsection{Relations entre les déterminants du choix de carrière et les caractéristiques sociodémographiques}

Un modèle d'équations structurales a été développé afin d'observer les relations entre les variables. Notons que les variables «statut conjugal», «niveau de formation professionnelle supérieure», «niveau de formation professionnelle inférieure» et «nombre d'années d'encadrement d'apprentis» ne sont pas significativement corrélées avec les déterminants du choix de carrière; par conséquent, nous ne les avons pas retenus dans cette analyse. Les sept variables sociodémographiques (région linguistique, âge, taux d'activité, sexe, parentalité, niveau de formation académique et le nombre d'années d'expérience en tant qu'enseignant) ont été introduites comme 
Les déterminants du choix de carrière des enseignants de la formation professionnelle...

prédicteurs des douze déterminants du choix de carrière (perception des capacités à enseigner, choix par opportunité, valeur intrinsèque de la carrière, utilité personnelle, utilité sociale, expériences passées d'enseignement et d'apprentissage, influences sociales, dissuasion sociale, carrière d'expert, forte demande, statut social et moral des enseignants et salaire).

Le modèle final, après suppression des pistes non significatives, s'ajuste adéquatement aux données $\left(n=483 ; \chi^{2}{ }_{(55)}=62.77, p=.22\right)$. Dans ce modèle, les variables sociodémographiques expliquent de $1 \%$ (motivation intrinsèque) à $19 \%$ (carrière de spécialiste) de la variance dans les divers déterminants du choix de carrière. Les résultats figurent dans le tableau 3. 
Tableau 3

Résultats de l'analyse en pistes causales: effets des caractéristiques sociodémographiques sur les déterminants du choix de carrière

\begin{tabular}{|c|c|c|c|c|c|c|c|c|c|c|c|c|}
\hline $\begin{array}{l}\text { caractéristiques } \\
\text { sociodémographiques }\end{array}$ & PCE & $\mathrm{COP}$ & VIC & UTP & UTS & EXP & INS & DIS & CEX & FDE & SME & SAL \\
\hline $\begin{array}{l}\text { Région }(0=\text { Romandie, } 1= \\
\text { Suisse alémanique })\end{array}$ & - & $\begin{array}{l}-0.268^{\mathrm{c}} \\
(0.041)\end{array}$ & - & $\begin{array}{l}-0.140^{\mathrm{a}} \\
(0.063)\end{array}$ & - & - & $\begin{array}{l}-0.205^{\mathrm{c}} \\
(0.047)\end{array}$ & $\begin{array}{l}-0.145^{b} \\
(0.053)\end{array}$ & $\begin{array}{l}0.364^{\mathrm{c}} \\
(0.069)\end{array}$ & - & $\begin{array}{l}0.145^{\mathrm{b}} \\
(0.045)\end{array}$ & - \\
\hline Âge & - & - & - & $\begin{array}{l}-0.160^{b} \\
(0.056)\end{array}$ & - & $\begin{array}{l}-0.182^{c} \\
(0.042)\end{array}$ & - & $\begin{array}{l}-0.157^{b} \\
(0.049)\end{array}$ & - & - & - & - \\
\hline $\begin{array}{l}\text { Taux d'activité }(0=\text { accessoire, } 1 \\
=\text { principal })\end{array}$ & $\begin{array}{l}0.119^{b} \\
(0.043)\end{array}$ & - & $\begin{array}{c}0.105^{\mathrm{a}} \\
(0.043)\end{array}$ & $\begin{array}{l}0.138^{\mathrm{a}} \\
(0.063)\end{array}$ & - & $\begin{array}{c}0.096^{\mathrm{a}} \\
(0.045)\end{array}$ & - & - & - & $\begin{array}{l}0.216^{\mathrm{c}} \\
(0.048)\end{array}$ & - & $\begin{array}{l}-0.111^{\mathrm{a}} \\
(0.048)\end{array}$ \\
\hline Sexe $(0=$ homme, $1=$ femme $)$ & - & - & - & - & $\begin{array}{l}-0.121^{b} \\
(0.040)\end{array}$ & $\begin{array}{l}-0.125^{\mathrm{a}} \\
(0.049)\end{array}$ & $\begin{array}{l}-0.193^{c} \\
(0.043)\end{array}$ & - & $\begin{array}{l}0.255^{\mathrm{c}} \\
(0.066)\end{array}$ & $\begin{array}{l}0.183^{b} \\
(0.053)\end{array}$ & - & - \\
\hline Parentalité & - & - & - & $\begin{array}{l}0.232^{\mathrm{c}} \\
(0.065)\end{array}$ & - & - & - & - & - & - & - & - \\
\hline $\begin{array}{l}\text { Niveau de formation académique } \\
(0=\text { autre niveau de formation, } 1 \\
=\text { niveau de formation } \\
\text { académique })\end{array}$ & - & $\begin{array}{l}-0.208^{\mathrm{c}} \\
(0.045)\end{array}$ & - & - & - & - & - & $\begin{array}{l}0.140^{\mathrm{b}} \\
(0.053)\end{array}$ & - & - & $\begin{array}{l}-0.129^{b} \\
(0.041)\end{array}$ & - \\
\hline $\begin{array}{l}\text { Nombre d'années d'expérience } \\
\text { en tant qu'enseignant }\end{array}$ & - & - & - & - & - & - & - & - & - & - & - & $\begin{array}{l}0.136^{\mathrm{c}} \\
(0.039)\end{array}$ \\
\hline $\mathrm{R}^{2}$ & 0.014 & 0.096 & 0.011 & 0.117 & 0.015 & 0.058 & 0.077 & 0.068 & 0.191 & 0.065 & 0.044 & 0.029 \\
\hline
\end{tabular}

Notes: a $\mathrm{p}<.05 ; \mathrm{b} \mathrm{p}<.01 ; \mathrm{c} \mathrm{p}<.001$. Abréviations: $\mathrm{PCE}=$ Perception des capacités à enseigner; $\mathrm{COP}=\mathrm{Choix}$ par opportunité; VIC $=$ Valeur intrinsèque de la carrière; UTP $=$ Utilité personnelle; UTS $=$ Utilité sociale; EXP = Expériences passées dans l'enseignement et l'apprentissage; INS = Influences sociales; DIS = Dissuasion sociale; CEX = Carrière d'expert; FDE = Forte demande; SME = Statut social et moral des enseignants; SAL = Salaire. Les variables «Statut conjugal», «Niveau de formation professionnelle supérieure», «Niveau de formation professionnelle inférieure» et «Nombre d'années d'encadrement d'apprentis» ne se trouvent pas dans le tableau, car elles n'ont pas d'effet significatif sur les déterminants de choix de carrière. 
En premier lieu, concernant les motivations, la perception des aptitudes à enseigner est prédite par la filière de formation destinée aux enseignants exerçant leur activité à titre principal $(\beta=.12, \mathrm{p}<.01)$. La région linguistique (alémanique) $(\beta=.27, p<001)$ et un niveau de formation académique $(\beta=-.21, p<.001)$ sont associés négativement à «choix par opportunité». Pour ce qui concerne «valeur intrinsèque de la carrière», seul le taux d'activité la prédit significativement $(\beta=.11$, $\mathrm{p}<.05)$. Par contre, «utilité personnelle» est prédite par de nombreuses caractéristiques individuelles: négativement par la région linguistique (alémanique) $(ß=-.14, p<.05)$ et l'âge $(\beta=.16, p<.01)$ et positivement par la filière de formation destinée aux enseignants exerçant l'activité à titre principal $(\beta=.14, \mathrm{p}<.05)$ et la parentalité $(\beta=.23, \mathrm{p}<.001)$. Concernant «utilité sociale», celle-ci est prédite négativement par le fait d'être de sexe féminin. «Expériences passées dans l'enseignement et l'apprentissage» est négativement prédite par l'âge $(\beta=-.18$, $\mathrm{p}<.001)$ et le sexe féminin $(\beta=-.13, \mathrm{p}<.05)$, mais au contraire positivement par le taux d'activité principale $(\beta=.10, \mathrm{p}<.05)$. Ensuite, les femmes $(\beta=-.19, \mathrm{p}<.001)$ de la région alémanique $(B=-.20, \mathrm{p}<.001)$ seraient moins influencées socialement dans leur choix. Dans la même composante «influences de socialisation», les personnes de Suisse alémanique $(\beta=-.21, \mathrm{p}<.001)$ ainsi que l'âge $(\beta=-.19$, $\mathrm{p}<.001$ ) prédisent négativement «dissuasion sociale» alors que le niveau de formation académique la prédit positivement $(\beta=.14, \mathrm{p}<.01)$.

Pour ce qui est des perceptions du métier d'enseignant, «salaire» est prédit positivement par le nombre d'années d'expérience en tant qu'enseignant ( $\beta=.14$, $\mathrm{p}<.001)$, mais négativement par la filière à taux d'activité principale $(\beta=-.11$, $\mathrm{p}<.05)$. Cette même variable $(\beta=.22, \mathrm{p}<.001)$, logiquement, ainsi que le sexe féminin $(\beta=.18, \mathrm{p}<.01)$ prédisent positivement la perception de «forte demande». Les personnes de la région alémanique $(\beta=.15, \mathrm{p}<.01)$ perçoivent «statut social et moral des enseignants» comme étant plus haut alors que le niveau de formation académique a un effet inverse sur cette perception $(\beta=-.13, \mathrm{p}<.01)$. Finalement, la région linguistique allemande et le sexe féminin prédisent fortement la perception de l'enseignement comme «carrière d'expert» $(\beta=.36, \mathrm{p}<.001$ et respectivement $\beta=.26, p<.001)$. 


\section{Discussion}

Pour rappel, l'objectif de cette étude était de déterminer, à l'aide d'une adaptation du modèle théorique FIT-Choice développé par Watt et Richardson (2007) les motivations qui amènent les enseignants en formation professionnelle à ce deuxième choix de carrière ainsi que leurs perceptions de cette profession. De plus, des analyses ont été réalisées pour déterminer le lien entre les motivations et perceptions des enseignants et leurs caractéristiques sociodémographiques.

\subsection{Prépondérance relative des déterminants du choix d'une carrière enseignante chez les enseignants de la formation professionnelle}

Nos analyses démontrent que «valeur intrinsèque de la carrière», «perception des aptitudes à enseigner» et «utilité sociale»sont les raisons les plus invoquées pour expliquer le choix de devenir enseignant. En d'autres termes, les enseignants de la formation professionnelle mettent davantage en évidence les facteurs liés à l'intérêt pour le métier, le sentiment d'efficacité dans la profession et l'envie de contribuer socialement et de travailler avec les jeunes comme étant déterminants dans leur choix de transiter de la pratique à l'enseignement. A l'inverse, les motivations de type personnel telles «salaire» ou «temps pour la famille» ne sont rapportées que peu fréquemment dans ce même choix.

Ces conclusions confirment les observations d'un certain nombre de recherches menées ces dernières années (Bruinsma et Jansen, 2010; Chin et Young, 2007; Huberman, 1989; Watt et Richardson, 2007) avec des enseignants du niveau secondaire. En effet, les enseignants de la formation professionnelle semblent, en regard de ces différentes recherches, également privilégier les motivations altruistes ou intrinsèques aux motivations extrinsèques (Kyriacou et Coulthard, 2000). En d'autres termes, il ne semble pas y avoir de différences dans la nature des motivations des enseignants à entrer dans la profession selon le niveau de formation dans lequel ils sont actifs. 
Les déterminants du choix de carrière des enseignants de la formation professionnelle...

Un autre élément observé dans cette étude est la relative faiblesse de l'importance des facteurs sociaux («influences sociales» et «dissuasion sociale»), à l'exception de la dimension «expériences passées dans l'enseignement et l'apprentissage» qui marque les individus et pourrait les amener, bien des années plus tard, à choisir la profession d'enseignant corroborant ainsi les observations de Lortie (1975/2002). Finalement, comme l'ont souligné également Nägele et Bestvater (2012) et Deschenaux et Roussel (2008), le motif «choix par opportunité» joue un rôle non négligeable dans le choix d'une carrière dans l'enseignement, ce qui semble démontrer que tous les enseignants ne parviennent pas dans ce métier par vocation.

Concernant les perceptions, nos analyses soulignent que les enseignants considèrent leur métier comme exigeant («carrière d'expert» et «forte demande»), mais dont on peut également retirer des bénéfices («salaire» et «statut social et moral des enseignants»). Toutefois, les enseignants jugent leur profession comme étant plus exigeante que ce qu'elle n'apporte de bénéfices, ce qui semble indiquer que la transition vers l'enseignement n'est pas un choix professionnel réalisé par facilité.

\subsection{Relations entre les caractéristiques sociodémographiques des enseignants et l'importance qu'ils accordent aux divers déterminants du choix de carrière}

Trois caractéristiques sociodémographiques ressortent particulièrement des analyses comme prédictifs des déterminants de choix de carrière: la région linguistique (culture), le taux d'activité et le sexe. Les coefficients $\beta$ observés sont relativement faibles, ce à quoi nous nous attendions de par la nature de ces prédicteurs. De même, la variance expliquée $\left(\mathrm{R}^{2}\right)$ varie entre $1 \%$ («valeur intrinsèque de la carrière» et «perception des capacités à enseigner») et $19 \%$ concernant «carrière d'expert».

Les enseignants de la région francophone seraient plus enclins à débuter dans le métier par opportunité ou à la suite d'influences sociales, guidés par des motivations davantage liées aux valeurs d'utilité personnelle, alors que les enseignants de la partie alémanique de la Suisse attribuent un statut social plus élevé au métier et considèrent 
Les déterminants du choix de carrière des enseignants de la formation professionnelle...

plus fortement que ce dernier exige une haute expertise de spécialiste. Les raisons de ces différences entre les deux régions linguistiques sont à chercher, du moins en partie, dans les différences de valeur accordée à la formation professionnelle dans ces deux parties de la Suisse. Ainsi, les motivations plus sociales et volontaires des enseignants de la région alémanique pourraient être dues à une valorisation plus forte et un ancrage culturel plus profond de la formation professionnelle dans cette région qu'elle ne l'est dans la partie francophone du pays (Forschungsprogramm UNIVOX, 2004/2005). Il est intéressant de constater que les différences culturelles et linguistiques en Suisse jouent ici un rôle significatif dans les déterminants de la transition de la pratique à l'enseignement. Les recherches ne se sont encore qu'insuffisamment penchées sur ce facteur.

Un statut professionnel d'enseignant à un taux d'activité principale prédit des valeurs plus élevées dans les «perceptions des capacités à enseigner». Ces enseignants relèvent également avoir vécu des expériences passées dans l'enseignement et l'apprentissage plus positives et ne pas considérer le salaire comme une motivation importante, ce qui ne les empêche cependant pas de rapporter davantage de motivations à valeur d'utilité personnelle. Ceci montre que les enseignants à un taux d'activité principale arrivent au métier pour des raisons qui se veulent sociales sans que cela n'exclue des raisons plus personnelles. En d'autres mots, ils seraient davantage habités par des motivations quel que soit leur ordre.

Les femmes sont significativement moins influencées par l'entourage social et par leurs expériences passées dans l'enseignement et l'apprentissage et auraient par contre des motivations ayant davantage trait aux valeurs d'utilité personnelle que les hommes. Ils se pourraient que ce processus soit lié au fait que les femmes soient davantage concernées par la recherche d'un travail à temps partiel que les hommes. Elles considèrent également que l'enseignement exige une plus grande charge de travail et un statut d'expert.

Globalement, ces résultats semblent indiquer que les enseignants de la partie alémanique parviendraient à l'enseignement davantage par «vocation» que ceux de la partie francophone et qu'une personne qui fait le choix de l'enseignement pour des raisons d'utilité personnelle («temps pour la famille», «sécurité de l'emploi», etc.) 
aurait le type de l'individu de sexe féminin, vivant dans la partie francophone de la Suisse, plutôt jeune, suivant une formation destinée aux enseignants exerçant l'activité à titre principal et parent d'un ou plusieurs enfants.

\subsection{Implications}

Au vu de la problématique des effectifs d'enseignants dans les pays occidentaux, les résultats obtenus dans cette étude pourraient apporter certaines informations supplémentaires utilisables notamment lors de la planification de stratégies de recrutement de nouveaux enseignants. Les moyennes obtenues (comparables à celles des enseignants au niveau secondaire) semblent démontrer qu'il est judicieux de repérer les candidats ayant des motivations intrinsèques et d'utilité sociale plutôt que ceux qui ont des motivations d'utilité personnelle, ces derniers démontrant un engagement professionnel plus faible (Sinclair, 2008) et un risque plus élevé d'abandonner leur carrière dans l'enseignement (Huberman, 1989; Müller et al., 2009).

Si les motivations les plus favorables à rencontrer chez les candidats sont connues, il n'empêche que les analyses plus détaillées menées selon les caractéristiques sociodémographiques démontrent qu'il ne serait pas inutile d'adapter les pratiques de recrutement en fonction du sexe, du taux d'activité et (dans le cas d'un contexte plurilingue et pluriculturel) de la région d'origine notamment. En d'autres termes, il serait envisageable d'attirer par exemple davantage de femmes en mettant en exergue les possibilités du travail à temps partiel ou, plus spécifiquement dans notre contexte, se servir davantage de l'image valorisée de la formation professionnelle auprès des hommes de la région alémanique.

Les données recueillies peuvent être une potentielle source d'informations précieuse pour les formateurs pour la planification des offres de formation. Ceci ouvre la voie à d'enrichissantes collaborations entre les milieux de la recherche et ceux de la formation dans une perspective pluridisciplinaire. Depuis quelques années, des efforts sont fournis dans ce sens dans le contexte de la formation professionnelle en Suisse. 
Les déterminants du choix de carrière des enseignants de la formation professionnelle...

À la lumière de cette recherche, un des enjeux dans la lutte contre la pénurie des enseignants semble résider dans divers facteurs. Premièrement, dans l'équilibre à trouver entre la mise en valeur des motivations intrinsèques et d'utilité sociale dans le but d'attirer dans l'enseignement des candidats qui s'engagent et perdurent dans le métier. Deuxièmement, dans l'adaptation des politiques de recrutement par la mise en évidence d'avantages de différents ordres en fonction des caractéristiques sociodémographiques des candidats. Troisièmement, dans la prise en considération des motivations et perceptions des enseignants lors de la planification des contenus de formation, ceci dans un cadre propice à la collaboration entre chercheurs et formateurs.

\subsection{Limites de la recherche et perspectives}

Ces résultats doivent être considérés à la lumière des limites de notre recherche. Tout d'abord, l'enquête demandait aux participants d'autoévaluer et de rapporter leurs motivations et perceptions et les réponses pourraient ainsi être biaisées par un phénomène de désirabilité sociale au moment, notamment, de répondre aux questions ayant trait aux motivations d'utilité personnelle, ceci malgré l'anonymat garanti dans cette enquête. Une autre limite qui est induite par le moment auquel les enseignants en formation professionnelle ont été interrogés est apparue du fait que ces derniers avaient déjà un certain nombre d'années d'expérience dans l'enseignement avant de s'engager dans leur formation et de participer à notre étude. Ainsi nous pouvons faire l'hypothèse que ces années de pratique ont influencé les représentations des enseignants concernant leurs motivations à enseigner. Finalement, nous n'avons pas considéré l'intérêt personnel ou l'enthousiasme pour le domaine professionnel dans notre étude alors qu'il pourrait constituer un déterminant non négligeable pour les enseignants de notre étude (Kunter, Tsai, Klusmann, Brunner, Krauss et Baumert, 2008; Long et Woolfolk Hoy, 2006).

Un élément que nous n'abordons pas dans cette étude, mais qui pourrait constituer une prochaine étape dans la compréhension du métier d'enseignant, serait de mieux saisir le rôle prédictif que peuvent avoir les motivations sur le 
développement et l'évolution professionnelle des enseignants durant leur formation et leur carrière (Huberman, 1989) et plus précisément leur engagement professionnel (Sinclair, 2008), leurs perceptions et connaissances pédagogiques (Crahay, Wanlin, Issaieva et Laduron, 2010) et leur sentiment d'efficacité à enseigner (Bandura, 1997). Pouvoir déterminer ainsi le rôle des motivations sur ces différents facteurs liés à la pratique enseignante permettrait également de mieux cibler les contenus de formation, d'améliorer le recrutement des enseignants et peut-être également de diminuer le taux de retrait de ceux qui y sont entrés. Méthodologiquement, cela implique certainement des recherches longitudinales faisant appel également à des récoltes de données qualitatives afin de comprendre par quels processus la formation influence le développement et l'évolution des motivations et perceptions des enseignants en formation professionnelle.

\section{Références}

Achtenhagen, F. et Grubb, W.N. (2001). Vocational and occupational education: Pedagogical complexity, institutional diversity. In V. Richardson (Éd.), Handbook of research on teaching (p. 604-639). Washington, DC: American Educational Research Association.

Balleux, A. (2011). L'entrée en enseignement professionnel au Québec: un long parcours de transition en tension entre le métier exercé et le métier enseigné. Recherches en éducation, 11, 55-66.

Balleux, A. et Perez-Roux, T. (2011) (Éd.). Transitions professionnelles et recompositions identitaires dans les métiers de l'enseignement et de l'éducation. Recherches en éducation, 11.

Bandura, A. (1997). Self-efficacy: The exercise of control. New York: Freeman.

Beaucher, C. et Balleux, A. (2010) (Éd.). L'entrée en enseignement professionnel: images d'une réalité et questions de formation. Nouveaux c@hiers de la recherche en éducation, 13(1).

Berger, J.-L. et D'Ascoli, Y. (2011). Les motivations à devenir enseignant: revue de la question chez les enseignants de première et deuxième carrière. Revue française de pédagogie, 175, 113-146.

Berger, J.-L. et D'Ascoli, Y. (2012a). Becoming a VET teacher: Investigating the determinants of career choice and their relation to perceptions about prior occupation. Asia-Pacific Journal of Teacher Education, 40(3), 319-343 (Special 
Les déterminants du choix de carrière des enseignants de la formation professionnelle...

issue: Teaching Motivations in Different Countries: Comparisons using the FITChoice scale (Guest editors: H.M.G. Watt et P.W. Richardson).

Berger, J.-L. et D'Ascoli, Y. (2012b). Motivations to become Vocational Education and Training educator: A Person-oriented approach. Vocations et Learning, 5(3), 225-249.

Bollen, K.A. (1989). Structural equations with latent variables. New York: Wiley.

Bruinsma, M. et Jansen, E.P.W.A. (2010). Is the motivation to become a teacher related to pre-service teachers' intentions to remain in the profession? European Journal of Teacher Education, 33(2), 185-200.

Centre suisse de coordination pour la recherche en éducation. (2010). L'éducation en Suisse: rapport 2010. Aarau: SKBF/CSRE.

Chin, E. et Young, J.W. (2007). A person-oriented approach to characterizing beginning teacher in alternative certification programs. Educational Researcher, $36(2), 74-83$.

Conférence suisse des directeurs cantonaux de l'instruction publique (2008). Profession enseignante: analyse des changements et conclusions pour l'avenir. Berne: Auteur.

Crahay, M., Wanlin, P., Issaieva, E. et Laduron, I. (2010). Fonctions, structuration et évolution des croyances (et connaissances) des enseignants. Revue française de pédagogie, $172,85-129$

Deschenaux, F. et Roussel, C. (2008). L'accès à la carrière enseignante en formation professionnelle au secondaire: le choix d'un espace professionnel. Nouveaux c@hiers de la recherche en éducation, 11(1), 1-16.

Deschenaux, F. et Roussel, C. (2011). L'expérience de métier: le catalyseur des conceptions de l'enseignement en formation professionnelle au Québec. Recherches en éducation, 11, 15-26.

Duchesne, C. (2011). Effectuer une transition professionnelle pour donner du sens à la vie. Recherches en éducation, 11, 27-38.

Dubs, R. (2006). Rapport d'expertise sur les questions concernant la formation professionnelle en Suisse. Berne: HEP Verlag.

Eccles, J.S. (2009). Who am I and what am I going to do with my life? Personal and collective identities as motivators of action. Educational Psychologist, 44(2), 78-89.

Eccles, J.S., Adler, T.F., Futterman, R., Goff, S.B., Kaczala, C.M., Meece, J.L. et Migdley, C. (1983). Expectancies, values, and academic behaviors. In J.T. Spence (Éd.), Achievement and achievement motives. Psychological and sociological approaches (p. 75-146). San Francisco, CA: W.H. Freeman. 
Enders, C. K. (2006). Analyzing structural equation models with missing data. In G.R. Hancock et R.O. Mueller (Éds.), Structural equation modeling: A second course (p. 313-342). Greenwich, CT: Information Age Publishing.

Eurydice. (2009). Key data on education in Europe 2009. Bruxelles: EURYDICE.

Forschungsprogramm UNIVOX (2004/2005). L'école et la politique éducative sous l'œil critique des citoyens suisses. Zürich: gfs et Hutmacher, W.

Gavillet-Mentha, F. (2011). Un métier désenchanté. Parcours d'enseignants secondaires. Lausanne: Antipodes.

Grollmann, P. (2008). The quality of vocational teachers: Teacher education, institutional roles and professional reality. European Educational Research Journal, 7(4), 535-547.

Hackett, G. (1995). Self-efficacy in career choice and development. In A. Bandura (Éd.), Self-efficacy in changing societies (p. 232-258). Cambridge, Royaume-Uni: Cambridge University Press.

Hof, S., Strupler, M. et Wolter, S.C. (2011). Quereinsteiger in den Lehrberuf am Beispiel der schweizerischen Berufsbildung [Les enseignants de deuxième carrière: étude de cas basée sur le système de formation professionnelle en Suisse.] Leading House Working Paper No. 59. Swiss Leading House on Economics of Education, Firm Behavior and Training Policies. Berne.

Huberman, M. (1989). La vie des enseignants. Lausanne: Delachaux et Niestlé.

Jellab, A. (2008). Sociologie du lycée professionnelle: L'expérience des élèves et des enseignants dans une institution en mutation. Toulouse, France: Presses universitaires du Mirail.

Kunter, M., Tsai, Y.-M., Klusmann, U., Brunner, M., Krauss, S. et Baumert, J. (2008). Students' and mathematics teachers' perceptions of teacher enthusiasm and instruction. Learning and Instruction, 18(5), 468-482.

Kyriacou, C. et Coulthard, M. (2000). Undergraduates' views of teaching as a career choice. Journal of Education for Teaching, 26(2), 117-126.

Long, J.F. et Woolfolk Hoy, A. (2006). Interested instructors: A composite portrait of individual differences and effectiveness. Teaching and Teacher Education, 22, 303-314.

Lortie, D.C. (1975/2002). Schoolteacher. A sociological study. Chicago: The University of Chicago Press.

Maroy, C. (2008). Perte d'attractivité du métier et malaise enseignant. Le cas de la Belgique. Recherche et Formation, 57, 23-38. 
Les déterminants du choix de carrière des enseignants de la formation professionnelle...

Müller, K., Alliata, R. et Benninghoff, F. (2009). Attracting and retaining teachers: A question of motivation. Educational Management Administration et Leadership, 37(5), 574-599.

Muthén, L.K. et Muthén, B. O. (2007). Mplus User's Guide. Fifth Edition. Los Angeles, CA: Muthén et Muthén.

Nägele, C. et Bestvater, A. (2012). "The attractiveness of VET teacher profession in Switzerland”. In Berger, J.-L. (dir.), The choice to become a vocational teacher: Economical, psychological, and social determinants. Symposium tenu à la Conférence de la Société suisse pour la recherche en éducation, 2 au 4 juin, Berne, Suisse.

Office fédéral de la formation professionnelle et de la technologie (2011). La formation professionnelle en Suisse: Faits et données chiffrés. Berne: Auteur.

Office fédéral de la statistique suisse. (2010a). Corps enseignant selon le degré d'enseignement et personnel des hautes écoles. Neuchâtel: Auteur.

Office fédéral de la statistique suisse. (2010b). Education statistics 2009. Neuchâtel: Auteur.

Organisation de coopération et de développement économiques (OCDE) (2005). Attracting, Developing and Retaining Effective Teachers. Paris: Auteur.

Organisation de coopération et de développement économiques (OCDE) (2009). Creating effective teaching and learning environments: First results from TALIS. Paris: Auteur.

Richardson, P.W. et Watt, H.M.G. (2010). Current and future directions in teacher motivation research. In T.C. Urdan et S.A. Karabenick (Éds.), The decade ahead: Applications and contexts of motivation and achievement (Advances in motivation and achievement, Vol. 16B, p. 139-173). Bingley, Royaume-Uni: Emerald.

Richardson, P.W. et Watt, H.M.G. (2006). Who chooses teaching and why? Profiling characteristics and motivations across three Australian universities. Asia-Pacific Journal of Teacher Education, 34(1), 27-56.

Richardson, P.W., Watt, H.M.G. et Tysvaer, N.M. (2007). What motivates people from business-related careers to change to teaching? In M. F. Ozbilgin et A. Malach-Pines (Éds.), Career choice in management and entrepreneurship: A research companion (p. 219-239). Cheltenham, Royaume-Uni: Edward Elgar Press.

Satorra, A. et Bentler, P.M. (2001). A scaled difference chi-square test statistic for moment structure analysis. Psychometrika, 66, 507-514.

Sinclair, C. (2008). Initial and changing student teacher motivation and commitment to teaching. Asia-Pacific Journal of Teacher Education, 36(2), 79-104. 
Les déterminants du choix de carrière des enseignants de la formation professionnelle...

Tabachnick, B.G. et Fidell, L.S. (2007). Using multivariate statistics ( $5^{\mathrm{e}}$ éd.). Boston, MA: Allyn et Bacon.

Watt, H.M.G. et Richardson, P.W. (2007). Motivational factors influencing teaching as a career choice: Development and validation of the FIT-Choice scale. The Journal of Experimental Education, 75(3), 167-202.

Watt, H.M.G. et Richardson, P.W. (2008). Motivations, perceptions, and aspirations concerning teaching as a career for different types of beginning teachers. Learning and Instruction, 18, 408-428. 
Les déterminants du choix de carrière des enseignants de la formation professionnelle...

\section{Annexe \\ Traduction et adaptation des échelles et items du modèle FIT-Choice}

(Watt et Richardson, 2007)

\section{Partie A. Motivation}

«J'ai décidé de devenir enseignant parce que...»

Perceptions des capacités à enseigner

Je dispose des aptitudes caractérisant un bon enseignant.

J'ai de bonnes compétences pour enseigner.

Le métier d'enseignant est en adéquation avec mes capacités.

Valeur intrinsèque de la carrière

Le métier d'enseignant m'intéresse.

J'ai toujours voulu être enseignant.

J'aime enseigner.

Enseigner m'enthousiasme.

\section{Opportunité}

L'opportunité d'enseigner s'est présentée.

Certaines circonstances favorables m'ont amené à l'enseignement.

J'ai simplement eu l'occasion d'enseigner.

\section{Utilité personnelle}

\section{Sécurité de l'emploi}

L'enseignement me permet de suivre une carrière stable.

L'enseignement m'assure un salaire fixe.

L'enseignement est un métier sûr.

\section{Temps pour la famille / Facilité de l'emploi}

Enseigner à temps partiel me permet d'avoir plus de temps pour ma famille.

Les heures d'enseignement sont en adéquation avec les responsabilités familiales.

Les vacances scolaires permettent de remplir les obligations familiales.

En tant qu'enseignant, j'ai de longues vacances.

En tant qu'enseignent, mes journées de travail seront courtes.

\section{Utilité sociale}

\section{Améliorer l'équité sociale}

L'enseignement me permet d'élever les ambitions des jeunes défavorisés.

L'enseignement me permet d'œuvrer pour les personnes défavorisées.

Influencer le futur des enfants/adolescents / Réaliser une contribution sociale

L'enseignement me permet de transmettre certaines valeurs aux jeunes.

L'enseignement me permet d'exercer une certaine influence sur les générations futures.

L'enseignement me permet de rendre un service à la société.

Les enseignants fournissent une contribution essentielle à la société.

Le fait d'enseigner me permet de rendre quelque chose à la société.

\section{Travailler avec des enfants/adolescents}


Les déterminants du choix de carrière des enseignants de la formation professionnelle...

Je souhaite travailler avec des jeunes.

Je souhaite exercer un métier axé sur les jeunes.

J'aime travailler avec les jeunes.

J'ai envie d'aider les jeunes à apprendre.

\section{Expériences passées dans l'enseignement et l'apprentissage}

J'ai eu des enseignants qui m'ont inspiré.

J'ai eu de bons enseignants en modèle.

J'ai vécu des expériences d'apprentissage positives.

Influences sociales

Mes amis pensaient que je devrais devenir enseignant.

Ma famille pensait que je devrais devenir enseignant.

Des personnes avec qui j'ai travaillé pensaient que je devrais devenir enseignant.

Dissuasion sociale

Vous a-t-on encouragé à suivre une autre carrière que l'enseignement?

Vous a-t-on dit que l'enseignement n'était pas un bon choix de carrière?

Vous a-t-on incité à envisager d'autres carrières que l'enseignement?

Vous a-t-on conseillé une autre carrière que l'enseignement?

\section{Partie B. Perceptions du métier d'enseignant}

\section{Carrière d'expert}

Pensez-vous que l'enseignement requière une expertise de haut niveau?

Pensez-vous que les enseignant-e-s doivent faire preuve d'un haut niveau de connaissances spécifiques?

\section{Forte demande}

Pensez-vous que les enseignant-e-s soient soumis à une lourde charge de travail?

Pensez-vous que l'enseignement soit exigeant sur le plan émotionnel?

Pensez-vous que l'enseignement soit difficile?

\section{Statut social et moral des enseignants}

Pensez-vous que les enseignant-e-s soient perçu-e-s comme des spécialistes?

Pensez-vous que l'enseignement soit perçu comme un métier ayant un haut statut?

Pensez-vous que la carrière d'enseignant soit respectée en tant que telle?

Pensez-vous que le moral des enseignant-e-s soit élevé?

Pensez-vous que les enseignant-e-s se sentent valorisé-e-s au sein de la société?

Pensez-vous que les enseignant-e-s estiment que leur métier bénéficie d'un haut statut social?

Salaire

Pensez-vous que l'enseignement soit un métier bien rémunéré?

Pensez-vous que les enseignant-e-s reçoivent un bon salaire? 